Article received on $11^{\text {th }}$ Jun 2014

Article accepted on $14^{\text {th }}$ Jun 2014

UDC: 786.2 .087 .5

\title{
Man-Ching Yu*
}

The Hong Kong Institute of Education Cultural and Creative Arts Department Musical Arts Division

\section{PITCH-CLASS ANALYSIS: SOME ASPECTS OF IC5 AND IC1 DESIGN IN GYÖRGY LIGETI'S PIANO ÉTUDES}

\begin{abstract}
This paper investigates pitch-class organization in György Ligeti's piano Études, focusing on the organizational design of particular interval classes. In the etudes, IC5 and IC1 are generative in constructing materials in different dimensions and at different surface levels. Additionally, the unfolding of pitch classes initiates ascending and descending circles of fifths, at small-scale and large-scale structural levels alike, illuminating the prominence of the fifth in Ligeti's work, which harks back to the commonpractice period.
\end{abstract}

Key words: Ligeti, interval class, pitch class

Ligeti's diverse musical style underwent various transformations during different periods; each period demonstrates substantive stylistic differentiation, along with his diverse compositional techniques. In particular, it is the textures and harmonic materials in association with particular intervallic structures ${ }^{1}$ that

\footnotetext{
* Author contact information: manchingyu@msn.com

${ }^{1}$ For instance, in his early and mature works, he used chromatic elements almost to exhaustion. See Miguel A. Roig-Francoli, "Harmonic and Formal Processes in Ligeti's Net-structure Compositions", Music Theory Spectrum, 1995, 17, 242-267.
} 
affect the resulting sound. The musical style of his earlier period generally reflects the influence of Bartók and Hungarian folklore. ${ }^{2}$ His post-1956 music is characterized by dense micropolyphonic webs. ${ }^{3}$ Besides micropolyphonic techniques, Ligeti used chromatic intervallic cells to generate a distinctive harmonic language, dubbed "harmonic net-structure". ${ }^{4}$ However, his late, post-1980 works generally feature experimenting with diatonic domains, as he noted that

[i]n the late '50s and early '60s my music moved within harmonic fields filled out with chromaticism, whereas ten years later I composed diatonic music. I felt that chromaticism had been exhausted: the choice was between going "back" to diatonic music and going "forward" outside the sphere of tempered Music. ${ }^{5}$

Among his late-period works, his Horn Trio of 1982 is the first piece where Ligeti experimented with his new style. ${ }^{6}$ In this piece, there is conspicuous use of major and minor harmonies (set class 3-11), reflecting Ligeti's exploration of triadic sonorities without any reference to the syntax of common-practice tonality. Along with set class 3-11, various diatonic set classes - 3-2 [013], 3-6 [024], 3-7 [025], and 3-11 [037], drawn from the diatonic collection 7-35 [013568A] - may be found in his later works. ${ }^{7}$ Moreover, Ligeti's particular applications

2 Among his works, Musica ricercata (1953) and String Quartet No. 1, Métamorphoses nocturnes (1953-1954) clearly manifest his earlier styles; see Michael D. Searby, Ligeti's Stylistic Crisis: Transformation in His Music Style, 1974-1985, Lanham, MD, Scarecrow Press, 2010.

${ }^{3}$ Micropolyphonic web is a technique adopted by Ligeti whereby numerous independent chromatic lines are intertwined with each other to create chromatic-filled complex sound clusters. It is evident in his large-scale orchestral works, such as Apparitions (1958-59) and Atmosphères (1961); see David Cope, Techniques of the Contemporary Composer, New York, Schirmer Books, 1997. Same here. Ligeti himself mentions tis important innovative concept in György Ligeti in Conversation with Péter Várnai, Josef Häusler, Claude Samuel, and Himself, London, Eulenburg, 1983, 13-82.

${ }^{4}$ Harmonic net structure is a continuous web of fine-woven lines in a constant interactive process of transformation of one or more parameters; his Ramification (1968-69) and String Quartet No. 2 (1968) exemplify this technique. Generally speaking, all types of pitch-class transformations exclusively exhibit chromatic properties in Ligeti's "net-structure" composition, and these include chromatic fluctuation as well as chromatic transformation of harmonic cells and triadic units; see Miguel A. Roig-Francoli, op. cit.

5 György Ligeti, Péter Várnai, Josef Häusler, and Claude Samuel, György Ligeti in Conversation with Péter Várnai, Josef Häusler, Claude Samuel and Himself, op. cit., 30-31.

${ }^{6}$ Ligeti himself confirmed that this horn trio was the first piece in his new style, belonging to the last period; see István Szigeti, “A Budapest Interview with György Ligeti”, The New Hungarian Quarterly, 1984, 25, 210.

7 See John D. Cuciurean, “Aspects of Harmonic Structure, Voice Leading, and Aesthetic Function in György Ligeti's in Zart Fliessender Bewegung, Contemporary Music Review, 2012, 31, 225. 
of trichordal set classes 3-7 $[025]^{8}$ and 3-11 $[037]^{9}$ were regarded as a sort of typical signals ${ }^{10}$ in his late works, and serve as generative harmonic devices for generating diatonic sound. ${ }^{11}$

There is emphasis on IC5 already in some of Ligeti's earlier works. For instance, in Hora lunga, the first free transformational ostinati start on $\mathrm{C}$ and end on $\mathrm{G},{ }^{12}$ forming an IC5 framework. Moreover, in his earlier Sonata for solo cello, IC5 likewise plays an equally prominent role, exclusively framing the melodic pitch-class structure of the piece. ${ }^{13}$ Besides the fact that IC5 plays a structural role in synthesizing various pitch classes, its interaction with IC1 as the benchmarked chromatic element in his earlier works is quite evident: Amy Bauer has observed that in Ligeti's Musica ricercata No. 11, the subjects are characterized by chromatic scales and their entrances relate to one another according to the circle of fifths. ${ }^{14}$ Moreover, in Bauer's own analysis of Ligeti's Lontano, in Canon $\mathrm{I}$, the perfect fourth and fifth, as ICs5 framing two diatonic segments, become an important signal in the second diatonic section, while chromaticism exclusively dominates the first section. In the second diatonic section, an IC1 spanning a major seventh between E4 and D\#5, serves as registral boundary, encompassing all pitches within. Along with Ligeti's earlier works featuring IC5 and IC1, some of his late works, including the Etudes, also imply this phenomenon. Clifton Cal-

${ }^{8}$ In fact, this particular diatonic set class [025] is pentatonic in nature, but there is no direct evidence that Ligeti was ever influenced by Chinese music. Nonetheless, in an interview he gave to Eckhard Roelcke in 2001, Ligeti spoke of pentatonicism as a feature of Chinese music; see Eckhard Roelcke, Träumen Sie in Farbe? György Ligeti im Gespräch mit Eckhard Roelcke, Vienna, Paul Zsolnay Verlag, 2003, 89.

9 Structurally speaking, set classes [025] and [037] are both framed by IC5 and this interval becomes a generative element for constructing various passages.

${ }_{10}$ See György Ligeti et al., op . cit., 29; the original interview was conducted in 1978. Cuciurean likewise confirms that sonorities generated from either conjoined 3-7 or 3-11 trichords are subsets or supersets of the diatonic collection 7-35, all of which assume an increasingly prominent role in Ligeti's so-called final period; see John. D. Cuciurean, op. cit., 226.

${ }^{11}$ In Ligeti's later works, set class 3-11 surfaces as a new Ligeti signal that begins to assume a more prominent role from the mid-1980s onward; see John. D. Cuciurean, op. cit., 225-226.

12 The underpinning of the melodic material with two pitches, $\mathrm{C}$ and $\mathrm{G}$, rivalled by $\mathrm{F}$, starts at the beginning of each ostinato figure, forming three tonal centres; see Benjamin Dwyer, "Transformational Ostinati in György Ligeti's Sonatas for Solo Cello and Solo Viola", in: Louise Duchesnau and Wolfgang Marx (eds.), György Ligeti: Of Foreign Lands and Strange Sounds, Woodbridge, Boydell Press, 2011, 19-52.

13 The tonality of the transformational ostinati throughout the piece is analogous to the tonic-dominant relationship; see Benjamin Dwyer, op. cit.

${ }^{14}$ See Amy Bauer, op. cit., 78. The entrances of the subject and countersubject are characterized by the following cycle: $\mathrm{E} \#-\mathrm{B} \#-\mathrm{G}-\mathrm{D}-\mathrm{A}$. 
lender makes an important observation regarding Étude No. 5, Arc-en-ciel: the initial harmonic progression at the beginning of the piece, $\mathrm{C}^{+}{ }^{7} \rightarrow \mathrm{C}^{-}{ }^{7}$, involves the operation of sliding the upper fifth from $\{\mathrm{E}, \mathrm{B}\}$ to $\{\mathrm{E} b, \mathrm{~B} b\} .{ }^{15}$ Obviously, this results from the interaction between the two IC5 dyads and moving by a half-step (IC1). The next progression, from $\mathrm{C}^{-7}$ to $\mathrm{E}^{+}{ }^{7}$ likewise involves an IC5 operation: transposing the lower fifth up by a perfect fifth or moving the lower fifth one step up along the circle of fifths, $\{C, G\}$ to $\{G, D\}$, whilst holding the shared notes $\{\mathrm{E} b, \mathrm{~B} b\}$. Another Étude, Vertige, is characterized by endlessly descending chromatic lines, but due to their canonic treatment, there are chords involving fluctuation between seven and five semitones. Thus, it is evident that IC $5^{16}$ plays a structural role there as well, even though there is much chromaticism. ${ }^{17}$ Moreover, in his Étude Désordre, Ligeti juxtaposes a pentatonic F\# collection with a diatonic $\mathrm{C}$ collection, resulting in a totally chromatic effect. ${ }^{18} \mathrm{As}$ we can see, the work of perfect fifths and semitones is palpable in the Etudes. Furthermore, no one has investigated in depth the pitch-class organization of IC5 and IC $1{ }^{19}$ in Ligeti's late works, and it would be worthwhile to examine the organizational design of IC5 in the Études, in particular its interaction with IC1. My current investigation focuses only on the Études, as they constitute an important set of piano works in Ligeti's late oeuvre; 20 they show the wider stylistic changes of the 1980s as well as a more determined independence from the avant-

${ }^{15}$ Clifton Callender, "Interaction of the Lamento Motif and Jazz Harmonies in György Ligeti’s Arc-en-ciel", Intégral, 2007, 21, 1-23.

${ }^{16}$ In my earlier paper on Ligeti's White on White, the results of my analysis suggest that the harmonic materials are exclusively characterized by the consonant interval IC5, contributing to the transformational properties of the piece; see Man-Ching Donald Yu, "Transformations of Diatonic Materials and Tonal Procedures in Ligeti's Etude No. 15, White on White", New Sound, 2013, 41, 117-138.

17 See Manfred Stahnke, "The Hamburg Composition Class", in: Louise Duchesnau and Wolfgang Marx (eds.), op. cit., 223-244.

${ }_{18}$ Amy Bauer, op. cit.

19 Recently, interaction between IC5 and IC1 as well as the roles of fifths and semitones have been addressed in the music of Shostakovich and Copland; see Stephen C. Brown, "Ic1/Ic5 Interaction in the Music of Shostakovich", Music Analysis, 2011, 28/1, 185-220; David Heetderks, "A Tonal Revolution in Fifths and Semitones: Aaron Copland's Quiet City", Music Theory Online, 20111, 17/2; and Stanley V. Kleppinger, "Copland's Fifths and Their Structural Role in the Sonata for Violin and Piano", Music Theory Online, 2011, 17/2. Focusing on investigating the pitch-class organization of a particular composer's works seems an interesting topic for theorists. Thus, my research here might provide another way of looking at Ligeti's music, especially given that no one has thoroughly investigated the organizational use of these particular interval classes in his music.

${ }^{20}$ The Études reflect Ligeti's 40 years of compositional experience; see Richard Steinitz, György Ligeti: Music of the Imagination, Boston, Northeastern University Press, 2003. 
garde, whilst retaining a more personal connection with tradition. ${ }^{21}$ Second, I will also explore the formational role of the diatonic collection in association with tonal procedures predicated on the circle of fifths, ${ }^{22}$ to show the manner oof organization in which successive pitch-classes unfold. These two issues will be investigated at various levels, ranging from foreground to background levels, as well as vertical and horizontal dimensions, throughout a number of the Études. Additionally, horizontal and diagonal lines (indicating IC5 and IC1) connecting note heads without beams will serve as schematic representation ${ }^{23}$ of pitch-class organization, delineating the interrelationship between different pitch classes that highlights the importance of IC5 and IC1.

\section{The Interrelationship between IC5 and IC1 in Ligeti's Earlier Works}

To begin, Ligeti's emphasis on IC5 has already been highlighted in his Sketch, ${ }^{24}$ as shown in Example 1. Ligeti uses four perfect fifths and these particular intervals serve as central arrival points, wherefrom various chromatic dyads unfold. In fact, this simulated unfolding process from IC5 to IC1 and vice versa is easy to discern in one of his earlier works, the Invention for solo piano (1948).

Example 1: Page I of the Sketch for Bewegung, emphasizing perfect fifths

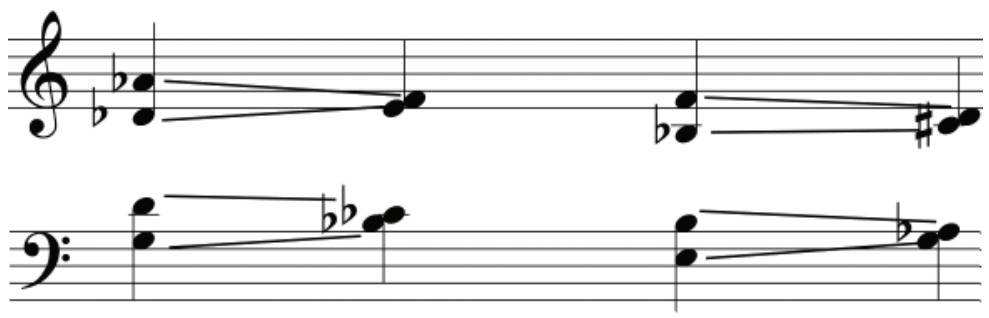

${ }^{21}$ Richard Steinitz, op. cit., 2003.

22 Inessa Bazayev has addressed the circle of fifths in Russian non-tonal music, in particular in works by Roslavets and Stravinsky; see Inessa Bazayev, "The Circle of Fifths in Russian Non-tonal Music: A Case Study of Selected Works by Roslavets and Stravinsky", Dutch Journal of Music Theory, 2013, 18/2, 52-68.

${ }^{23}$ Although I use schematic representation, it implies no Schenkerian concepts from the analytical point of view.

${ }^{24}$ See John D. Cuciurean, op. cit. 
Example 2: The unfolding and organization of fifths and semitones in Invention (1948)

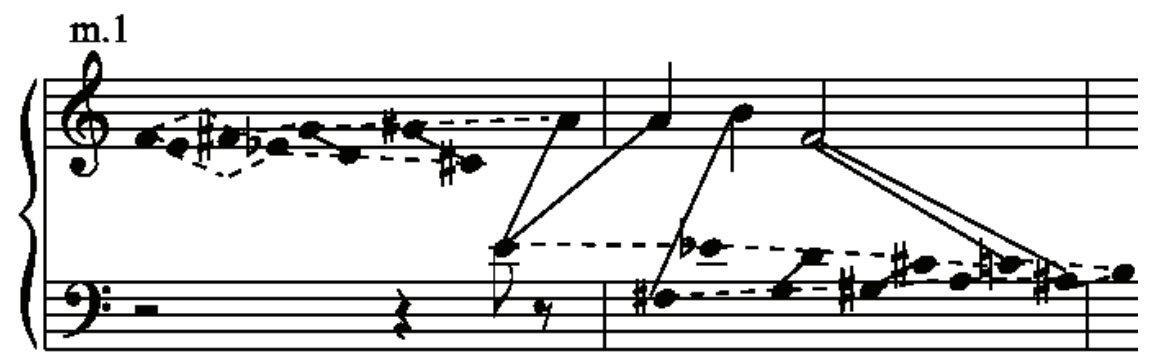

Example 2 illustrates the unfolding of pitch classes in bb. $1-3^{25}$ of the Invention, showing the main subject. The dotted lines indicate semitonal paths, while the solid ones indicate perfect-fifth relationships. In the right hand, the IC1 chromatic dyad with PCs 5/4 \{F4, E4 $\}$ at the beginning unfolds in contrary motion; the upper-layer F4 ascends chromatically, by four semitones, until it reaches A4; the lower-layer E4 descends chromatically, by three semitones, until it reaches $\mathrm{PC} 1$. Subsequently, the initial chromatic dyad transforms into two IC5 dyads with PCs0/7 $\{\mathrm{C} 4, \mathrm{G} 4\}$, followed by PCs1/8 $\{\mathrm{C} \# 4, \mathrm{G} \# 4\}$. The stability of IC5 is continually preserved by PC 4, E4 - the first pitch in the left hand, and PC9, A4 - the final pitch in the right hand in b. 1. On the first beat of b. 2, PC6, F\#3 (the left hand) maintains an IC5 link with PC11, B4 (the second beat in the right hand), while two ICs5 continue to maintain PCs $2 / 7$ (D4, G3\} and PCs $1 / 8\{\mathrm{C} \# 4, \mathrm{G} \# 3\}$ in the left hand, each PC unfolds in its own layer, in contrary motion. Consequently, before reaching the final pitch, PC11, B4, two ICs5 conclude the sentence: PC5, F4 in the right hand is juxtaposed with $\mathrm{PCs} 0 / 10\{\mathrm{C} 4, \mathrm{A \#} 3\}$ in the left hand. Furthermore, the middle section of the piece reveals an interesting phenomenon: stacked IC5 trichords successively interlinked by IC5 and IC1 across both layers. The first trichord $\{\mathrm{E} b 3, \mathrm{~A} b 3$, $\mathrm{D} b 4\}$ undergoes a $\mathrm{T} 5$ transformation into the second trichord $\{\mathrm{Bb} 4, \mathrm{~Eb} 5, \mathrm{Ab} 5\}$, and then a descending T11 transformation into the third $\{\mathrm{B} 3, \mathrm{E} 4, \mathrm{~A} 5\}$. The interlink between IC5 and IC1 is thus evident and these two intervals later serve as prominent agents in conglomerating the pitch classes in the Etudes.

In one of his other earlier "harmonic net-structure" works, Continuum (1968), IC5 reflects its pitch-class organizational significance during the transformational process. ${ }^{26}$ Materials are transformed either by adding another pitch

${ }^{25}$ Again, for the sake legibility, only the note heads are shown and most of the remaining examples here follow the same mode of presentation.

26 "Transformational process" here refers to the concept addressed by Roig-Francoli in 
Example 3: The interlink between IC5 and IC1 in Invention (1948)

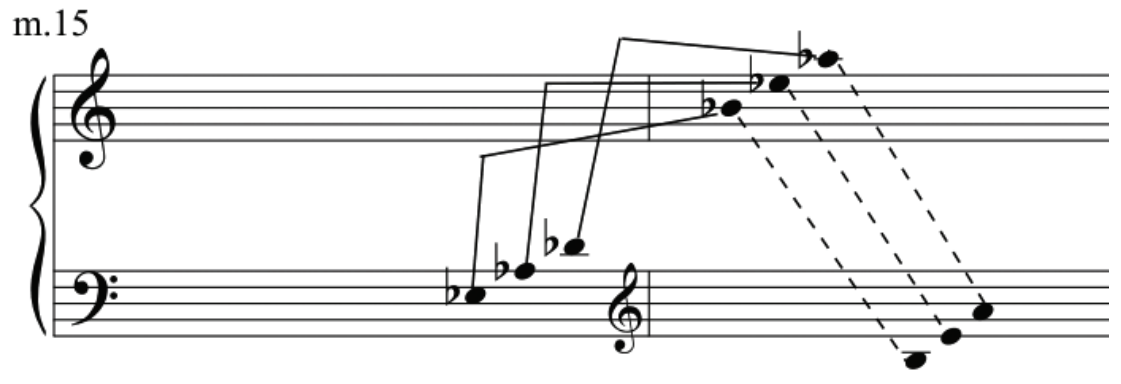

class or by means of chromatic inflection. Example 4 illustrates overall transformational processes from Block a through e. ${ }^{27}$ The music begins with Block a and transforms into a three-note cell (Block b) with the insertion ${ }^{28}$ of PC5, F4. This cell occurs 41 times, which is more than any of the remaining transformed cells (Blocks c through e). Block b, which constitutes set class [025], is an important diatonic agent that Ligeti emphasizes in his late works. Ligeti preserves this set class in Block c by inserting PC9, A4. This additive procedure is particularly interesting as the chromatic quality of the IC1 between PCs 9 and $10\{\mathrm{~A} 4, \mathrm{~B} b 4\}$ tends to counterbalance the consonant IC5 between PCs 5 and $10\{\mathrm{~F} 4, \mathrm{~B} b 4\}$. In Block d, PC 5, F4 is transformed by chromatic inflection into PC6, F\#4, resulting in two IC1 intervals; however, the chromatic-oriented quality in Block $\mathrm{d}$ is further counterbalanced by the addition of PC11 ( $\mathrm{C} b 4)$ in Block e, forming another IC5 between PCs 6 and $11\{\mathrm{~F} \# 4, \mathrm{C} b 4\}$, encompassing three ICs 1 inside the five-note cell.

\section{Example 4: IC5 and IC1 serving as intervallic agents}

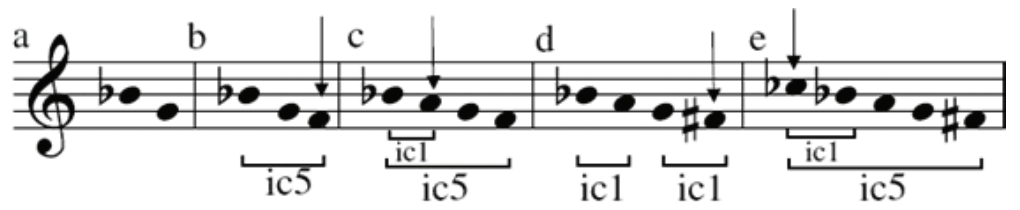

1995: pitch-classes are inserted for displaying intervallic symmetry among different chromatic cells.

${ }^{27}$ Each block represents a repetitive cell throughout the piece and each one is repeated numerous times, before transforming into the next block. For the sake of convenience, I have schematically notated each particular repetitive cell for each block, as shown in Example 4. ${ }^{28}$ Insertions of additional pitches are marked by arrows, as in Example 4. 
Example 5: The transformation of the pentatonic set class [027]

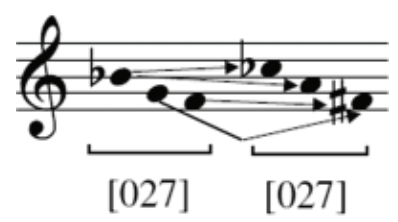

Example 5 shows a condensed version of the transformation of the trichord [025] (Block b) at a larger-scale surface level. The second trichord in Example 5 comprises the pitch classes mutated or added during the course of the transformation from Blocks $\mathrm{b}$ to e. In the first trichord, $\mathrm{PC} 10, \mathrm{~B} b 4$ transforms into PCs 11 and $9\{\mathrm{Cb} 4, \mathrm{~A} 4\}$ by contrary motion, whereas PCs 7 and $5\{\mathrm{G} 4, \mathrm{~F} 4\}$ transform downward and upward into PCs 9 and $6\{\mathrm{~A} 4, \mathrm{~F} \# 4\}$;

F4 transforms upward into PC 6. Two different members of SC [027] exhibit four IC1 chromatic inflections by means of contrary motion between different voices. Despite the chromatic transformation, the consonant quality is maintained by framing IC5 between the top and bottom voices of the two members. As is evident, Ligeti maintains the consonant stability of IC5 during the "net-structure" transformational process. In some of his late works, IC5 plays substantive generative roles and in the remainder of this paper, I shall focus on some passages from the Études. More often than not, materials at the beginning and end reveal some interesting phenomena.

\section{Interaction between IC5 and IC1 in Ligeti's Piano Études}

Having established the interrelationship between IC5 and IC1 in Ligeti's earlier works, I will turn now to the Etudes. I will begin by examining the pitchclass organizational role of IC5 and IC 1 at the beginning and end of passages drawn from various Études: a rather microscopic surface level will be addressed at first, followed by a discussion of the organizational role of these two intervals at a larger-surface level. Example 6a shows the opening thematic material, which is made up of two overlapping SCs, [016] and [015], in the right and left hand, respectively. ICs 5 and 1 form the core of these two set classes as both contain IC1 and IC5. Moreover, four ICs1 dominate the passage: $\{\mathrm{F} 4, \mathrm{E} 4\}$, $\{\mathrm{B} 4, \mathrm{C} 4\},\{\mathrm{E} 5, \mathrm{~F} 3\}$, and $\{\mathrm{F} 5, \mathrm{E} 3\}$. The constant non-linear emergence of ICs 1 is amalgamated with the linear-oriented emergence of ICs 5 across different layers, resulting in the interaction between the linear IC5 motion and the vertical IC1 sound, indicated by the diagonal dotted lines in Example 6 b.

Example 7 shows the opening of Vertige: pitch classes unfold linearly by semitonal shifts, while IC5 serves as registral boundary in both hands in each of the first four bars. In b. 1, PC11, B4 descends by a semitone until it reaches PC4, E4, whereupon the same sequence repeats and most importantly, IC5 between PCs11 and 4 \{B4, E4 $\}$ encompasses all pitch classes within. In b. 2, IC5 con- 
Example 6a: IC1-related SCs [015] and [016]

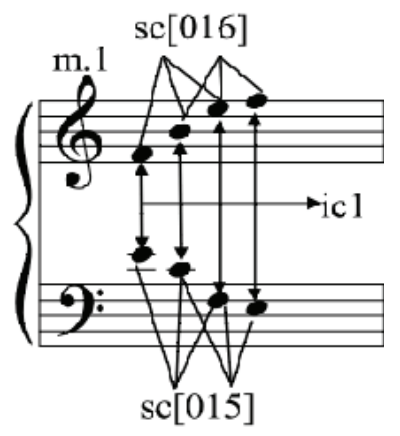

Example 6b: IC5 across two layers

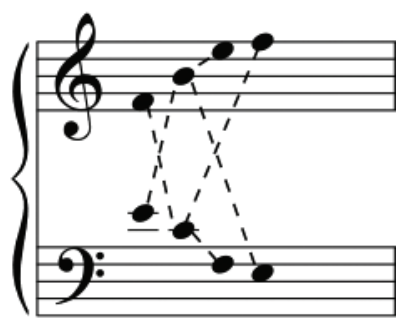

nects different pitch classes linearly: $\mathrm{PC} 3, \mathrm{E} b 4$ in the lower layer transforms ${ }^{29}$ into PC10, B 64 in the upper layer and so forth. In bb. 3 and 4, IC5 continues its overwhelming domination across both layers; however, it takes place vertically. Furthermore, at a larger surface level, in b. 3, the first pitch class, PC10, Bb4 in the right hand is in an IC5 relationship with the final pitch class, PC3, Eb4 in the left hand (all ICs5 are indicated by solid lines). Ligeti's pervasive use of IC5 and IC1 at the beginning of the passage illustrates the dichotomy between these two intervals - they are both more or less equally represented in each bar. In b. 2, both intervals cross linearly, and thus the interactional process takes place at the same time, but in bb. 3-4, interaction occurs linearly and vertically alike.

\section{Example 7: IC5 network across both chromatic-oriented layers}

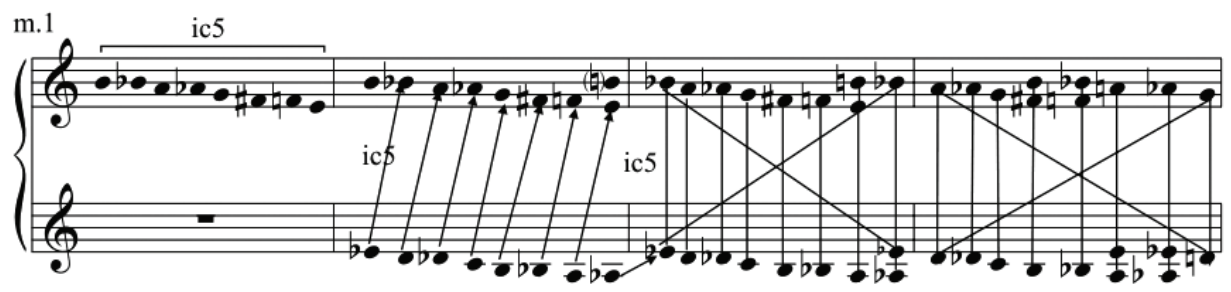

${ }^{29}$ The transformation to which I refer here indicates the linear motion of successive pitch classes. 
In Fem, Ligeti's use of IC5 and IC1 is highly visible, as shown in Example 8 . The roles of the two intervals highlight their significance by means of saturating both linear and vertical relationships between two layers right at the beginning of the piece. First, both layers are saturated with IC5 dyads and this highlights its intervallic prominence. Second, the importance of IC5 (indicated by the solid lines) is manifested by linear motion and simultaneous juxtapositions between the IC5 dyads both within each individual layer and between them. In $\mathrm{b} .1$, the perfect-fifth dyads $\{\mathrm{B} b, \mathrm{~F}\}$ and $\{\mathrm{A} b, \mathrm{E} b\}$ in the upper layer form an IC5 link with the IC5 $\{\mathrm{E} b, \mathrm{~B} b\}$ in the lower layer, proceeding by T7 and T5, respectively. Another T5 relationship is juxtaposed simultaneously between IC5 $\{\mathrm{A} b, \mathrm{E} b\}$ in the upper layer and IC5 $\{\mathrm{D} b, \mathrm{~A} b\}$ in the lower layer in the same bar; a successive T5 transformation appears from $\{\mathrm{D} b, \mathrm{~A} b\}$ to $\{\mathrm{Gb}$, $\mathrm{Db}\}$, as well as from $\{\mathrm{B}, \mathrm{F} \#\}$ and $\{\mathrm{E}, \mathrm{B}\}$ in $\mathrm{b}$. 2. In the same bar, $\{\mathrm{B} b, \mathrm{~F}\}$ in the upper layer descends by $\mathrm{T} 5$ to $\{\mathrm{F}, \mathrm{C}\}$, despite the insertion of $\{\mathrm{A} b, \mathrm{E} b\}$ in between. Along with IC5, IC1 is also amply represented in the interrelationship (indicated by the dotted lines) between individual dyads: in the lower layer, in b. 1, D5 and C5 form IC1 links with Db5, whilst moving in opposite directions, unfolding symmetrically to $\mathrm{D} b 5$. In $\mathrm{b}$. 1 , both the last $\{\mathrm{E} b, \mathrm{~B} b\}$ and the first dyad $\{\mathrm{B} b, \mathrm{~F}\}$ form linear IC1 links with the second dyad $\{\mathrm{E}, \mathrm{B}\}$ in $\mathrm{b}$. 2. Likewise in the lower layer, $\{\mathrm{B} b, \mathrm{~F}\}$ is juxtaposed with $\{\mathrm{B}, \mathrm{F} \#\}$ in an IC1 relationship. Thus, in the first two bars of this Etude, Ligeti establishes the prominence of IC 5 and IC1 by means of linear motion within and across layers, as well as by simultaneously juxtaposing them across individual layers.

\section{Example 8: The interrelationship of IC5 and IC1 across both layers}

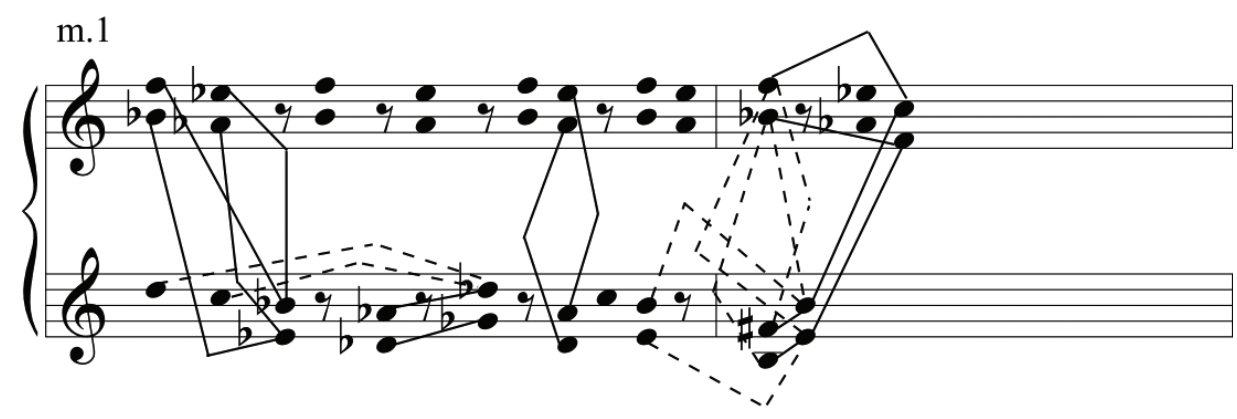

Examples 9a and 9b reflect the large-scale organizational IC5 and IC1 relationship between fifth-oriented dyads in the final section of Étude 16, Molto vivace. In the example below, all of the dyads are accented points in the passage and each is followed by a series of pitches. In Example 9a, the first, $\{A, D\}$, 
Example 9a: Large-scale organizational Example 9b: Large-scale organizational IC5 and IC1 linkage between successive IC1 interrelationship between the first dyads three dyads and the last dyad
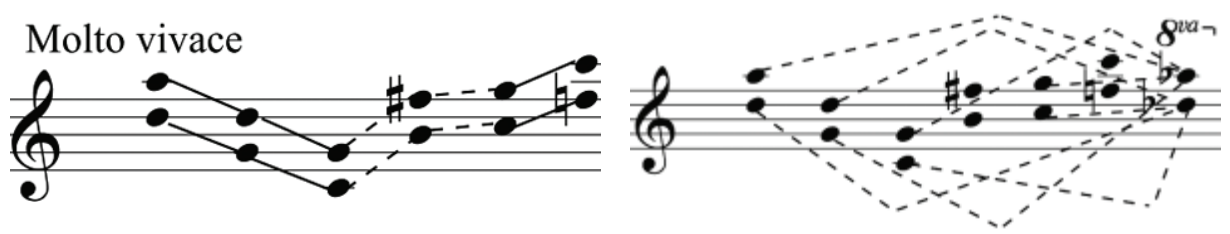

moves down by $\mathrm{T}^{30}$ to become $\{\mathrm{G}, \mathrm{D}\}$, which is then followed by another $\mathrm{T} 7$ to $\{\mathrm{C}, \mathrm{G}\}$. These three dyads exhibit three procedures stemming from the circle of fifths: A5-D5-G4-C4. ${ }^{31}$ The fifth dyad, $\{C, G\}$, is transposed up a perfect fourth, into $\{\mathrm{F}, \mathrm{C}\}$. Thus, IC5 relationships between these successive dyads are made apparent by descending $\mathrm{T} 7$ and ascending $\mathrm{T} 5$ transformations. On the other hand, the third dyad, $\{\mathrm{C}, \mathrm{G}\}$, transforms by an ascending $\mathrm{T} 11$ into $\{\mathrm{B}, \mathrm{F} \#\}$, followed by another fifth-dyad, $\{C, G\}$, up a T1. Example $9 \mathrm{~b}$ reveals another interesting phenomenon articulating the importance of IC1: those ICs1 are interlinked over a larger span with the final dyad $\{\mathrm{D} b, \mathrm{~A} b\}$; the first, second, third, and fifth dyads are respectively transformed by descending T1, ascending T13, and descending $\mathrm{T} 1,32$ ascending T13, and ascending $\mathrm{T} 1$. Thus, the prominent cadential role of the final dyad $\{\mathrm{A} b, \mathrm{D} b\}$ is interrelated with four different dyads emerging earlier, which illuminates the importance of $\mathrm{A} b 5$, related to the emphatic PC7 at the beginning of the Allegro vivace section. Here, the importance of IC1 is reflected at a larger-scale level, in which the emphasized pitches are interrelated, highlighting the interrelationship between different pitch centres in different sections (Example 10).

A similar instance in Automne à Varsovie likewise features a large-scale organizational event involving IC5 and IC1 operations. Example 11 shows prolonged pitch classes that arise during the course of the piece. ${ }^{33}$ Despite the fact that PC3 signals the beginning in b. 9 and marks the end of the piece in b. 98 ;

${ }^{30}$ Here I use ordered pitch-class intervals for addressing transposition in order to highlight the diversity of IC1 transformations.

31 This particular circle-of-fifths issue is even more pronounced in other passages, which are discussed below, in the second section of the paper.

${ }^{32}$ Here, the transformation is not induced by equivalent mapping (see Joseph N. Straus, Introduction to Post-tonal Theory, Upper Saddle River, NJ, Prentice Hall, 2005); G4 is transformed by an ascending T13 into Ab5 and D5 is transformed by a descending T1 into D $b 4$.

33 Although in the actual piece the repeated pitch classes more often appear in octave doublings, I have reduced them to a single pitch class each. In addition, the duration of the structural notes more or less shows the comparative length of each pitch class. 
Example 10: IC1 linkage with the accented pitch class, Ab6

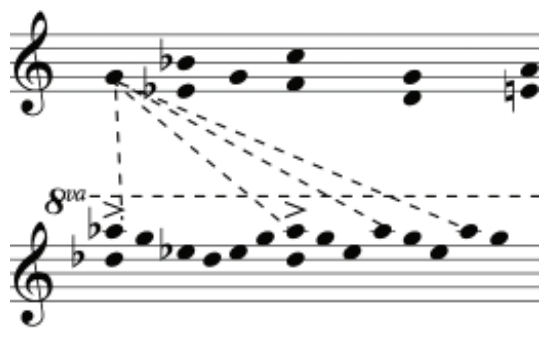

Example 11: A large-scale organizational event involving IC5 and IC1 operations as prolonged pitch centres

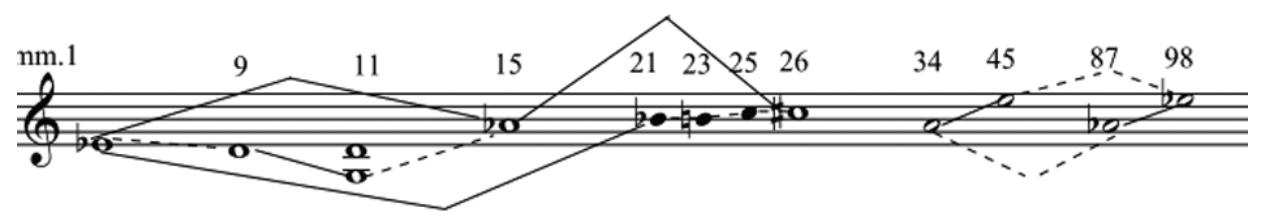

however, the two PCs3 are in an octave relationship. In b. 9, it transforms into PC2 by an IC1, adding a PC7 a fifth below, thus emphasizing two pitch classes in an IC5 relationship. In b. 15, PC8 is reinforced, forming an IC1 and IC5 relationship with PC7 and PC3, respectively. In bb. 1-15, those octave-oriented prolonged pitch classes are interrelated by the same two contrasting intervals, reflecting their significance in governing the background structural level of the piece. In bb. 21-25, the pitch classes are no longer octave-oriented until arriving at $\mathrm{PC} 1$ in b. 26. Although IC1 semitonal motion is prominent in governing the pitch centre, the first pitch class in this passage, PC10, is interlinked by IC5 with the opening pitch class of the piece, PC3 in b. 1; the fourth pitch class of the piece, PC1 is interlinked by IC5 with the fourth pitch class in b. 15, PC8. The mapping of the prolonged pitch classes in each section manifests the significance of IC5 and IC1 in each section. In bb. 34 and 45, a pair of IC5-oriented pitch classes, PC9 and PC4, emerges, and the pair is inflected by a semitone, generating PC8 in b. 87 and PC3 in b. 98, again forming a pair of IC5-oriented pitch classes.

The prominence of IC5 and IC1 also reflects their organizational significance with regards to chordal material. In the second movement of White on White, the final chord clearly manifests the superimposition of two IC5-oriented chords in an IC1 relationship (Example 12). The right hand contains a trichord consisting of two stacked perfect fifths, PCs1/6 $\{\mathrm{C} \# 6, \mathrm{~F} \# 5\}$ and PCs1/8 $\{\mathrm{C} \# 6, \mathrm{G} \# 6\}$, generating substantial dissonance with the trichord in the left hand, likewise comprising 
Example 12:

An IC1 superimposition of a chordal IC5

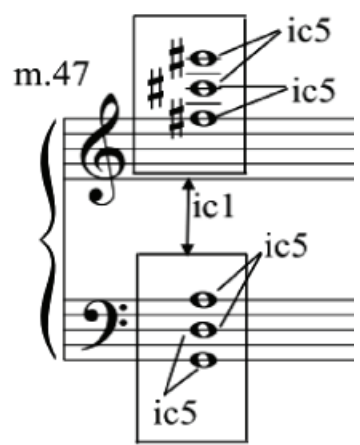

Example 13:

An IC1 transformation involving two chords

m. 90

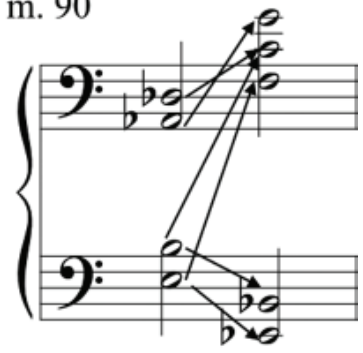

Example 14:

Simultaneous interaction of IC1 and IC5

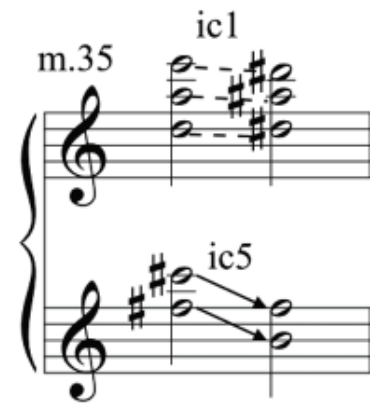

two stacked perfect fifths, with PCs $7 / 2\{\mathrm{G} 2, \mathrm{D} 3\}$ and PCs2/9 $\{\mathrm{D} 3, \mathrm{~A} 3\}$. Another IC1, between PC7 (G2, the lowest pitch class) and PC8 (G\#5, the highest pitch class) reinforces interaction between the two contrasting intervals.

At the end of Étude 12, Entrelacs, in b. 90, IC1 transformations cross between two IC5-oriented chords, as illustrated in Example 13. There are six instances of IC1 motion between different voices in different layers (indicated by arrows). In particular, B3, in the first chord, transforms doubly and symmetrically into PC0, $\mathrm{C} 4$ and $\mathrm{PC} 11, \mathrm{~B} b 2$; likewise, PC4, E3 undergoes a similar symmetrical transformation into PC5, F3 and PC3, Eb2. These massive instances of linear IC1 motion occur between two IC5-oriented chords, revealing the emphatic roles of these two intervals, albeit in different dimensions. Example 14 shows the last bar of Etude 11, En Suspens, illustrating another instance of interaction between IC5 and IC1 involving two stacked fifth chords. In this case, two different intervals move simultaneously: three instances of IC1 motion (dotted lines) in the upper layer and two instances of IC5 motion (solid arrows) in the lower layer. Judging from the examples above, Ligeti's use of IC5 along with IC1 appears to serve as an important benchmarking signal at the beginning and end of each piece.

\section{IC1 and the Circle of Fifths}

In addition to the prominence of IC5 and IC1 at various levels in the examples above, a number of the Etudes also feature the unfolding of the circle of fifths, at various structural levels. Often, a pitch class successively transforms into the next one by an IC5 in an organizational pattern and sometimes 


\section{Example 15: The insertion of semitone-inflected pitch classes outlining a descending circle of fifths in Capriccio No. 1}

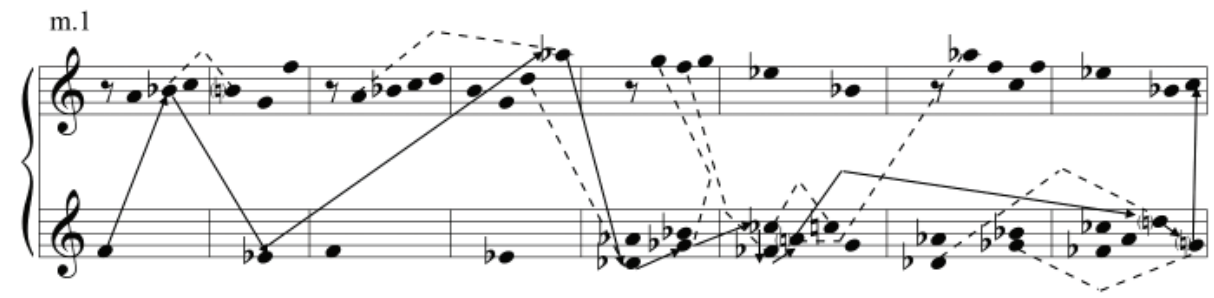

a chromatic-inflected pitch class governs the procedure. As a matter of fact, Ligeti used this procedure as early as the 1947 Capriccio No. 1, but in terms of compositional strategy, it becomes more interesting in the Études.

Example 15 shows bb. 1-8 from the Capriccio. The solid arrows indicate a descending circle of fifths: F4, right at the beginning, initiates a descending circle of fifths, connecting to $\mathrm{B} b 4$ in b. 1, proceeding to $\mathrm{E} b 4$ in b. 2, and then on to $\mathrm{A} b 5$ (b. 4), $\mathrm{D} b 4$ (b. 4), Gb4 (m. 4), Cb5 (b. 5), Fb4 (b. 5), A4 (b. 6), D5 (b. 8), G4 (b. 8), and finally C5 (b. 8), completing the cycle. It is noteworthy that all pitch classes in the passage emerge there for the first time, emphasizing the significance of their roles. Furthermore, their occurrences are triggered by semitonal inflections ( $\mathrm{B} b 4$ to $\mathrm{B} 4$ in bb. $1-2, \mathrm{C} b 5$ to $\mathrm{C} 5$ in b. 6, $\mathrm{G} b 4$ to $\mathrm{G} 4$ in bb. 7-8), an ascending T11 (A4 to Ab5 in bb. 6-7), descending T13 transpositions (D5 to $\mathrm{D} b 4$ in bb. 4-5, G5 to $\mathrm{G} b 4$ in b. 5, F5 to Fb4 in bb. 5-6), and an ascending T13 (Db4 to D5 in bb. 7-8), all of which contributes to the operation of IC1. Thus, in this early instance, Ligeti's use of the circle of fifths is evident, contributing to the operation of IC5, while the initial occurrences of those particular pitch classes are in turn initiated by the operation of IC1. Thus, in this instance, Ligeti's music features an intriguing network of intertwined operations of IC5 and IC1, which distinctly marks the pitch-class formation at the beginning of the piece.

Example 16 illustrates the large-scale transformational processes of Ligeti's Canon Etude, showing only the upper layer. ${ }^{34}$ The music begins with stacked diatonic fifths and pitch classes that gradually keep going "flat" are inserted in patterns drawn from the circle of fifths - F, $\mathrm{B} b, \mathrm{E} b, \mathrm{~A} b, \mathrm{D} b$, and $\mathrm{G} b$ - exhausting the entire pentatonic collection on PC6. On the other hand, other pitches are transformed by "sharps" and likewise placed into patterns drawn from the circle

${ }^{34}$ In the absence of bar lines, the rhythm is not indicated and in each layer, as shown in Example 6, pitch classes are presented as they occur in chronological order at a large-scale surface level. The emergence of the "flat-dominated" pitch classes is independent from the "sharp-dominated' pitch classes during the course of the piece. 
Example 16: Large-scale transformational processes in Canon along the circle of fifths, with semitonal networks spanning across both layers in various dimensions

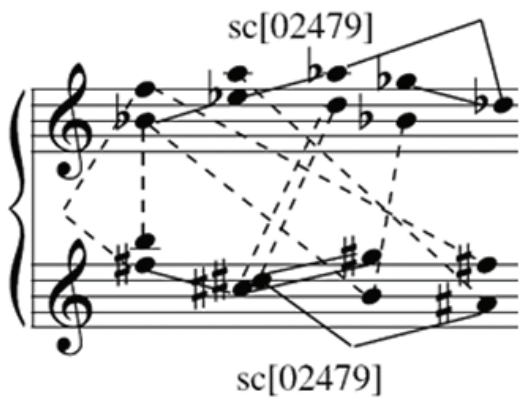

of fifths - B, F\#, C\#, G\#, D\#, A\#- gradually exhausting the entire pentatonic collection. Both collections contain the same pitchclasses, but their transformational processes are mutually inverted, as the "sharpening" circle of fifths starting on F\# progresses upward while the "flattening" circle of fifths starting on $\mathrm{B} b$ progresses downward. The dyads that trigger the transformational processes are in a semitonal relationship with each other and this initial IC1 relationship provides substantial contrast to the IC5-oriented large-scale transformational processes that occur during the course of the piece. More importantly, a large number of chromatic inflectional IC1 relationships occur between pitch classes belonging to different layers; even more importantly, both they and those pertaining to the circle of fifths occur the same number of times - nine. Viewed from a large-scale surface perspective, this strongly contributes to the unity of pitch-class organization in this piece. The IC5-IC1 network below suggests a large-scale link between the two contrasting intervals. This large-scale relationship between IC5 and IC1 may be viewed at the foreground level at the beginning of Étude 2, Cordes à vide, which is addressed below.

Another instance of interaction between IC5 and IC1 that also includes the circle of fifths is shown in Example 17 below. Right at the beginning of Cordes $a$ vide, ${ }^{35}$ Ligeti focuses solely on the circle of fifths; PC9, A5 initiates the procedure in the upper layer, while subsequent pitch classes outline a descending circle of fifths at the lower layer through the lowest pitch class, PC11, $\mathrm{Cb} 1$ in b. 2, completing the cycle with PC4, E5 back in the upper layer, i.e. $\mathrm{A} \rightarrow \mathrm{D} \rightarrow \mathrm{G} \rightarrow \mathrm{C} \rightarrow \mathrm{F} \rightarrow \mathrm{B} b \rightarrow \mathrm{E} b \rightarrow \mathrm{A} b \rightarrow \mathrm{D} b \rightarrow \mathrm{G} b \rightarrow \mathrm{C} b \rightarrow \mathrm{E}^{36}$ (indicated by arrows). Saturated with the IC5 procedure, upper voices feature semitonal shifts in the upper layer: $\mathrm{A} 5 \rightarrow \mathrm{B} b 5 \rightarrow \mathrm{B} 5$ and $\mathrm{D} 5 \rightarrow \mathrm{E} b 5 \rightarrow \mathrm{E} 5$ (indicated with dotted lines), resulting in a horizontalized linear interaction between the two domains. Moreo-

\footnotetext{
35 The piece is constructed on a succession of perfect fifths, but they sometimes occur simultaneously, sounding like open strings on a violin; see Richard Steinitz, op. cit., 286.

${ }^{36}$ Note that between $\mathrm{F}$ and $\mathrm{B} b$ and $\mathrm{C} b$ and $\mathrm{E}$, the pattern of descending perfect fifths breaks due to registral transfer; however, the circle of fifths is still there in the domain of pitch classes, disregarding the register of each pitch class, as different pitches are still connected by IC5.
} 
Example 17: Interactions between the circle of fifths and IC1 across layers

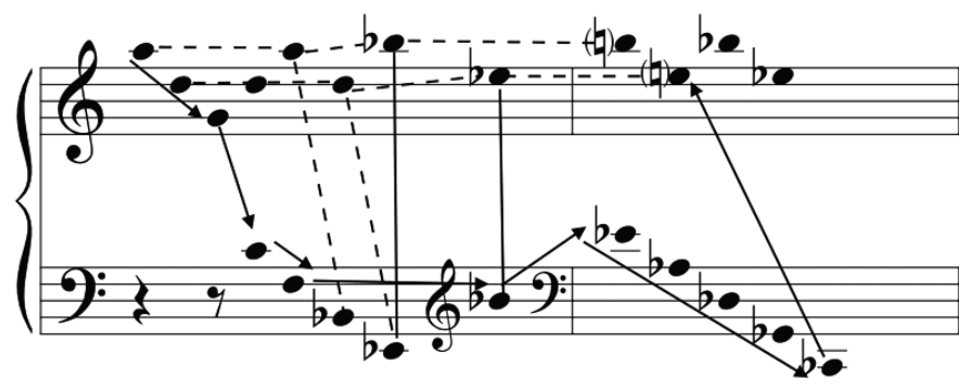

Example 18:

Pitch-class planning along the circle of fifths

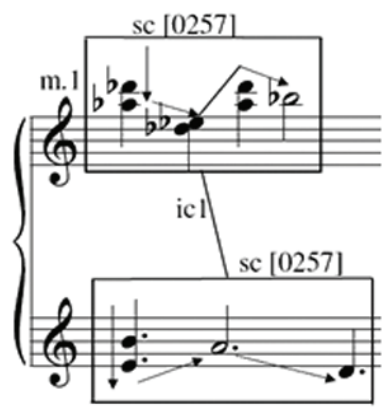

ver, IC1 emerges between the fifth and sixth pitch class, A5 and D5 in the upper layer, and between the third and fourth pitch class, $\mathrm{B} b 2$ and $\mathrm{E} b 2$ in the lower layer, generated by verticality rather than linearity. The dominance of IC5 verticality emerges between the seventh and eighth pitch class, $\mathrm{B} b 5$ and $\mathrm{E} b 5$ in the upper layer as well as between the fourth and fifth pitch class, Eb2 and $\mathrm{B} b 4$ in the lower layer. Both intervals occur the same number of times, reflecting a balanced utilization and interaction of IC5 and IC1. These two intervals serve as prominent intervallic agents for synthesizing the pitch-class formation right at the beginning.

Ligeti's use of the circle of fifths may be found in different diatonic territories. Example 18 shows the opening of En Suspens. The pitch-class organization between two layers is characterized by two different diatonic territories: the upper layer exhibits a black-note pentatonic scale on $\mathrm{G} b$, whereas the lower layer exhibits a white-note pentatonic scale on C. Ligeti juxtaposes two different sets of pentatonic materials, placing them in a semitonal IC1 relationship, as shown in Example 18. This chromatic relationship between the two pentatonic sets highlights the interaction between IC5 and IC1. The pentatonic set class [0257] in the lower layer stems from a descending circle-of-fifths sequence, $\mathrm{B} \rightarrow \mathrm{E} \rightarrow \mathrm{A} \rightarrow \mathrm{D}$, while the upper layer stems from an ascending circle of fifths sequence, $\mathrm{D} b \rightarrow \mathrm{A} b \rightarrow \mathrm{E} b \rightarrow \mathrm{B} b$, and their formational processes are headed in opposite directions. Ligeti establishes the pitch material of the piece right at the beginning by means of two different members of the pentatonic set class [0257], reflecting his strategic planning and emphasis on the perfect fifth in each layer. Moreover, there is interaction between the perfect fifth and IC1, as shown in 
Example 19a: A network of IC1 interactions between two pentatonic set classes

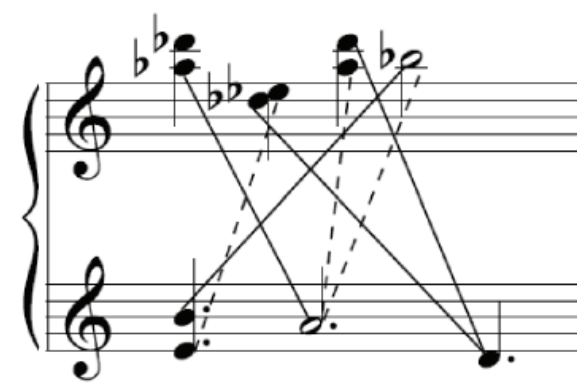

Example 19b: Interaction of ICs5 and 1 in vertical configuration

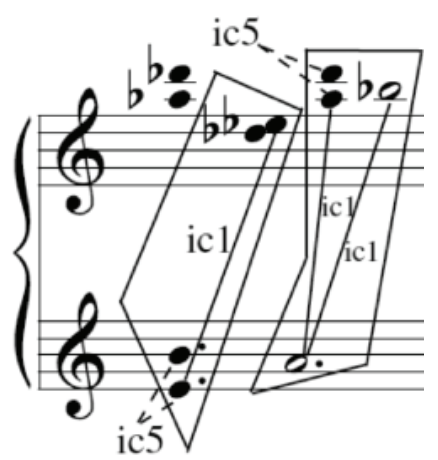

Example 19a. In the example, the dotted lines indicate that IC1 is generated by means of juxtaposing each individual pitch in each layer, whereas the solid lines indicate that the IC1 emerging from the succession of pitches is not reinforced simultaneously but rather cross linearly. The massive IC1 network counterbalances the perfect fifth, as shown in Example 19b.

Example 20 shows the opening of the central section, Allegro vivace, in Pour Irina. An ascending circle of fifths originates from Eb4 in an IC5 dyad $\{\mathrm{Eb}, \mathrm{Bb}\}$, proceeding both vertically and linearly (indicated by arrows) through the final B4. Thus, this circle of fifths procedure strongly links the four IC5 dyads, reflecting the double prominence of IC5. Moreover, aggregating all the pitch classes that stem from the circle of fifths generates a chromatic segment with set class [01235678T]; with the absence of G4, two chromatic groups (SC [0123]) may be formed by splitting the initial set class in two identical SCs. Subsequently, PC7 plays a central role in delineating the structure of the piece: in Allegro vivace, it emerges as the first pitch class and then again, after the first and fifth dyads. Furthermore, it forms an IC1 link with the accented PC Ab6, emphasized at the end of the piece, as I mentioned previously (Example 11). The passage features both linear and vertical interactions between IC5 and IC1. Linear ICs5 occur between Bb4 and F4, C5 and G4, D4 and A4, and E4 and B4, while linear ICs1 occur between Eb4 and D4, Eb4 and E4, and F4 and E4 at the lower pitch class of the IC5 dyad and another IC 1 occurs between Bb4/A4 and C5/B4 at the upper pitch class of the IC5 dyad. Thus, interaction between the two intervals takes place at different rates: the ICs5 along the circle of fifths occur faster than do the ICs1 between the pitch classes mentioned above.

Judging from the analysis and observations above, Ligeti's Etudes feature a systematic IC5 and IC1 design; more often than not, IC5 pervades the open- 
Example 20: Circle-of-fifths interlinks between IC5 dyads with chromatic motions

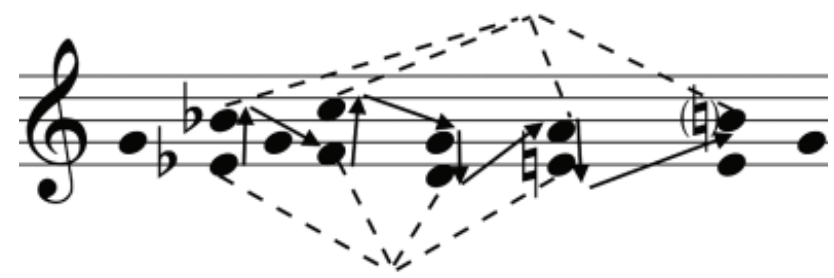

ing of a piece, blending with IC1 semitonal shifts and chromatic inflections for highlighting the contrasting quality of these two intervals. Moreover, these two intervals dominate a number of passages in various dimensions and surface levels, ranging from linear to vertical dimensions and small-scale to large-scale surface levels. Furthermore, IC5 is deployed along ascending and descending circles of fifths and this blends with IC1 and chromatic segments, to manifest the distinctive pitch-class organization of those passages - the link between IC5 and IC1. Although these phenomena are confined to the passages discussed above, one might infer that Ligeti's use of IC5 reflects his preference for consonant intervallic structures for the sake of departing from the sound of his earlier works; however, he preserves the most dissonant interval, IC1, from his earlier styles, serving as an agent for compromising the purity of the consonant IC5 in order to forge his multifaceted musical language. But one might ask, why did Ligeti tend to emphasize IC5 in his music? Perhaps because he wanted to dissociate himself from the chromatic sound of his earlier chromatic-oriented works. Moreover, it is the most consonant interval in the diatonic collection; the diatonic collection 7-35 [013569A] has the interval class vector [254361], in which IC5 occurs the maximum number of times and thus it is hardly surprising that IC5 plays such a central and generative role in some of his diatonic works that deserve our attention. Third, IC5 is the third consonant interval ${ }^{37}$ in the harmonic series; Ligeti's use of harmonic overtones is quite apparent in some of his works. It is possible that Ligeti takes advantage of this consonant interval for organizing the pitch material of his late diatonic works. Last, but not least, the appearance of the circle of fifths in Ligeti's music reflects his reference to the fifth relationship from the common-practice period, serving as a distinct way to organize pitch classes and refine his personal style by interacting and intermixing with chromatic elements.

${ }^{37}$ Here I specifically regard IC5 as the perfect $4^{\text {th }}$, but not the perfect $5^{\text {th }}$ in the harmonic series due to the ascending order of the series. 